\title{
Propuesta para mejorar la calidad en la práctica docente de los niveles medio y superior del IPN (México), a través de la formación del personal académico
}

\section{Proposal to improve the quality of teaching practice at the middle and high school levels of the IPN (Mexico), through the training of academic personnel}

DOI: $10.46932 / \mathrm{sjjdv2n2-023}$

Received in: january 1st, 2020

Accepted in: March 30th, 2020

\author{
Alejandro Mejía Carmona \\ Instituto Politécnico Nacional-México \\ E-mail: almejia@ipn.mx \\ Tiburcio Fernández Roque \\ Instituto Politécnico Nacional-México \\ E-mail: tfroque@yahoo.com.mx \\ José Arturo Correa Arredondo \\ Instituto Politécnico Nacional-México \\ E-mail: josearturocorrea@yahoo.com.mx \\ Jorge Sandoval Lezama \\ Instituto Politécnico Nacional-México \\ E-mail: jslezama09@yahoo.com.mx
}

\begin{abstract}
RESUMEN
El presente trabajo contiene un estudio descriptivo de los factores tales como; la inserción, trayectorias, permanencia y formación en la docencia; que intervienen en la profesionalización docente, en el contexto y marco normativo académico del Instituto Politécnico Nacional (IPN), México; con la intención de proponer un modelo de formación- profesionalización docente, basada en áreas de formación, competencias docentes y etapas de desarrollo profesional, con el objetivo de mejorar la calidad de la práctica docente en los niveles superior y medio superior del IPN.

Palabras clave: Calidad, mejoramiento, Competencias docentes, Formación docente.
\end{abstract}

\begin{abstract}
This paper contains a descriptive study of the factors such as; insertion, trajectories, permanence and teacher training; that intervene in teacher professionalization, in the context and academic regulatory framework of the National Polytechnic Institute (IPN), Mexico; with the intention of proposing a model of teacher training-professionalization, based on training areas, teaching competencies and stages of professional development, with the aim of improving the quality of teaching practice in the higher and middle levels of the IPN.
\end{abstract}

Key words: Quality, improvement, teaching competencies, teacher training. 


\section{INTRODUCCIÓN:}

Quienes ejercen la docencia en el IPN, llegaron a ella por diferentes motivos y circunstancias de vida. Hay quienes se formaron exprofeso para ello y hay quienes, de manera fortuita, azarosa o intencionada, optaron por ser docentes y descubrieron nuevas formas de ejercer una profesión, un oficio, un arte, un estilo de vida.

Cada docente desarrolla su labor educativa de diversas formas. En muchas ocasiones la ejercen por intuición; en otras, replican algunas metodologías de profesores que tuvieron cuando jóvenes, y en algunos casos más crean su propio estilo de docencia de manera autodidacta, o bien a partir del ámbito institucional.

Lo cierto es que, para quienes tienen la gran oportunidad de transmitir un conocimiento disciplinario a generaciones más jóvenes, tienen también la posibilidad de re-crear y re-inventar sus propios saberes y prácticas, así como el privilegio de coadyuvar en la formación profesional y académica de varias generaciones.

La experiencia de ser docente es única y a la vez compartida. Con la práctica, la experiencia construida y la voluntad de transformación, la docencia se convierte en una profesión que se estudia, se ejerce, se actualiza y se reconoce socialmente. Así, la labor del docente trae consigo una gran satisfacción, pero también un gran compromiso, pues son vidas con las que interactúa día con día, relación que tampoco es tan sencilla.

Si una persona desea ingresar al IPN para trabajar como docente, la admisión, nombramiento y adscripción, está descrita por el Reglamento de las condiciones interiores de trabajo del personal académico del Instituto Politécnico Nacional (IPN, 2018), en su capítulo VI, que destaca que "el aspirante a formar parte del personal académico del IPN, deberá cumplir con los siguiente requisitos: a) Ser de nacionalidad mexicana, o en el caso de extranjeros, contar con la autorización legal correspondiente para realizar el trabajo remunerado de que se trate; b) Comprobar haber realizado estudios completo en cualquier institución educativa nacional o extranjera, en la especialidad relacionada con el trabajo que va a desempeñar; c) Aprobar el concurso de oposición de cátedra correspondiente, d) Cumplir con las disposiciones y requisitos del presente reglamento, y demás aplicables al efecto".

Al analizar los aspectos anteriores, se observa que no existe un requisito de preparación mínima en aspectos relacionados con la docencia de los aspirantes a formar parte del personal académico. En consecuencia, la mayoría de los profesores que ingresa al IPN no tienen una formación docente a pesar de que la función primordial es la docencia.

Así pues, ya que la parte normativa no requiere que la persona que ingrese como personal académico al IPN tenga preparación docente y a pesar de que una de las funciones primordiales que 
ejercerá, será la docencia, se hace necesario desarrollar en el personal académico de recién ingreso y/o a los que no han participado en acciones de formación docente en el modelo por competencias.

Lo anterior con el propósito de ejercer una práctica docente consciente, mediante la reflexión e integración de elementos didácticos pedagógicos acordes al modelo educativo y académico del IPN y así contribuir a lograr el reto de construir una nueva cultura del trabajo académico que dinamice la docencia y su relación con la investigación y la extensión sobre la base de profesores con la formación idónea para el nivel que atienden.

Es por eso que este trabajo se convierte en una alternativa, que guie a las autoridades de las unidades académicas del IPN, responsables de la profesionalización docente en la parte de formación de competencias docentes, a través de la estrategia de proyectos y con base en un trabajo colaborativo, multinivel e interdisciplinar. El objetivo del presente trabajo, es ofrecer una propuesta de mejora de la práctica docente, para lograr una profesionalización de la labor docente que impacte directamente en forma positiva en el aula y en la formación integral de los alumnos del instituto.

\section{DESCRIPCIÓN DEL ESPACIO INSTITUCIONAL}

\subsection{DOCENCIA.}

En el Instituto Politécnico Nacional (IPN) la docencia se define como "el conjunto de actividades que el personal académico desempeña en el aula, el laboratorio, el taller; para planear, programar, llevar a efecto el proceso de enseñanza aprendizaje, conforme a los planes y programas de estudio aprobadas y de acuerdo con el programa de actividades que corresponden a su categoría académica. Además, el desarrollo de esta función incluye la preparación de clases, atención de alumnos, preparación de prácticas, aplicación y evaluación de exámenes”. En esta misma referencia el docente, se concibe como: "un miembro del personal académico, quien realiza actividades de docencia, de investigación científica y desarrollo tecnológico, de difusión de la cultura y las complementarias que se relacionan con las anteriores".

\subsection{EJERCICIO DE LA PRÁCTICA DOCENTE.}

El en el Instituto Politécnico Nacional (IPN, 1980), México, inicia con la selección y contratación por medio de un examen de oposición, cabe señalar que este proceso se implementó desde el surgimiento del IPN, en ese momento el modelo educativo era tradicional, después a finales de la década de los setentas, el modelo educativo se cambió a uno basado en la tecnología educativa y en 2003 se inició el cambio al modelo educativo basado en competencias, mismo que rige la educación que se imparte actualmente en los niveles superior y media superior. No obstante, los cambios del modelo 
educativo del IPN, el proceso de selección y contratación de los docentes permanecieron sin cambios. Este proceso consiste en una convocatoria para un examen denominado de oposición, en él se solicita a los aspirantes den una clase acerca de un tema incluido en las ahora llamadas unidades de aprendizaje para la que concursan, el jurado del examen les evalúa algunos aspectos pedagógicos todos referidos a lo que sería una exposición oral, tales como: facilidad para explicar, dicción, material para la exposición, conocimiento del tema, responde correctamente a las preguntas, entre otras; y esa es toda la exigencia pedagógica que se le pide para su contratación. No le son solicitados elementos didáctico-pedagógicos como: planeación didáctica, un plan de evaluación de aprendizajes, la elaboración de materiales didácticos, una rúbrica; entre otros muchos elementos que son indispensables para un ejercicio profesional de la docencia dentro de un modelo educativo por competencias. Esta manera de contratación de los docentes en el IPN, oculta tras de sí, el modo tradicional de ver al proceso enseñanza-aprendizaje, como simple transmisión de conocimientos en el que el alumno está vacío de conocimiento y hay que verterle información a través del discurso que después el deberá de reproducir textualmente. En este esquema el proceso de formación pedagógica del docente se considera que sucede durante el proceso mismo de la práctica docente, y es el caso de la formación docente que se da en el IPN.

\subsection{PROFESIONALIZACIÓN DOCENTE}

Para este trabajo, por profesionalización entenderemos por tal un proceso conducente a la construcción de una cierta profesionalidad. Abordando el tema de la profesionalidad docente, Desgagné (2005) nos enfrenta a la idea de su desarrollo bajo tres dimensiones: la identitaria, la ética y la reflexiva. La primera, hace alusión a la identidad docente, la que se construye en el tiempo y que sufre "shocks" biográficos que la transforman. La segunda, nos habla de la capacidad de los profesores de dar cuenta de sus actos y de dar respuestas éticas y pertinentes de los mismos. La tercera dimensión nos enfrenta al desafío de aprender de la experiencia, la que interpela nuestros conocimientos, creencias y valores. En estas tres dimensiones lo que aparece como desarrollo de la profesionalidad es, al entender de los autores del presente trabajo, el desarrollo de conciencia profesional docente, por lo que de ahora en adelante debiéramos pensar la profesionalización como un proceso permanente de concientización, cercano al concepto freiriano, y por tanto de liberación y transformación. En este sentido, el ejercicio profesional docente, asumido como un ejercicio de profesionalización, pasaría de la expresión de acciones y rutinas, a convertirse en una praxis, intencionada, generadora de sentidos y de saberes docentes. 


\subsection{CONCEPTO DE COMPETENCIA DOCENTE.}

Las competencias implican una nueva relación entre el docente-discente, nuevas formas de aprender, más que de enseñar, exigen una pedagogía diferenciada, nuevos métodos, trabajo colaborativo, interdisciplinar y multidisciplinar, un cambio definitivo en el quehacer docentes, esto requiere el desarrollo de nuevas competencias para los que decidan permanecer en este oficio. Las competencias docentes precisan profesionalización, práctica reflexiva, trabajo en equipo y por proyectos, autonomía y responsabilidad ampliadas, tratamiento de la diversidad, énfasis en los dispositivos y las situaciones de aprendizaje, sensibilidad con el conocimiento, prudencia con los procesos, estamos frente a nuevos escenarios para la función de "enseñar", lo cual implica un cambio de los roles tradicionales de maestroalumno, es este un escenario en formación. Por tanto, una función sustantiva de las instituciones educativas es el desarrollo de las competencias docentes, para ello será necesario instrumentar estrategias y programas que coadyuven al desarrollo de estas. En México, la Secretaría de Educación Pública, publica en octubre del 2008, el Acuerdo 447, en el que se establecen las competencias docentes para quienes impartan educación media superior (EMS) y educación superior (ES) en la modalidad escolarizada, estipula que las competencias docentes son las que formulan las cualidades individuales, de carácter ético, académico, profesional y social que debe reunir el docente de la EMS, y consecuentemente definen su perfil. Para este trabajo, se adopta de Garduño Rubio (2004), las competencias docentes pueden ser definidas como la forma práctica en que se articula el conjunto de conocimientos, creencias, capacidades, habilidades, actitudes, valores y estrategias que posee un docente y que determina el modo y los resultados de sus intervenciones pedagógicas. Cabe aclarar que para desarrollar competencias profesionales en las instituciones de educación superior se están redefiniendo las competencias docentes, ya que su tarea en este tipo de formación es "gestionar el ambiente de aprendizaje, interpretar las expresiones y comportamientos del educando y comunicarse con él para apoyar su proceso de aprendizaje. Este tipo de ejercicio docente es diferente al que se apega al guion de enseñanza de una disciplina para exponer su contenido y por ello se requiere de una preparación del docente acorde con lo que se requiere de él" (Chang, 2009).

\subsection{COMPETENCIAS PROFESIONALES DEL DOCENTE.}

En cuanto a los requerimientos de la práctica educativa en las instituciones de educación superior, el papel del docente en el desarrollo de las competencias profesionales (véase Perrenoud en el tabla1) tiene múltiples implicaciones de tipo integral y se basa en un modelo sistémico e interdisciplinario. De tal manera, el docente -en su saber, saber hacer y querer hacer- promueve el desarrollo de competencias profesionales. De ahí, la necesidad de definir las competencias docentes que garanticen la motivación del 
Tabla 1.- Lista de las competencias docentes.

\begin{tabular}{|c|c|c|c|c|}
\hline \multirow[t]{2}{*}{$\begin{array}{l}\text { Autor } \\
\text { País }\end{array}$} & $\begin{array}{l}\text { Rosa Ma. Esteban } \\
\text { Moreno (2011). }\end{array}$ & $\begin{array}{l}\text { Francisco de Asís Blas } \\
(2007) .\end{array}$ & $\begin{array}{l}\text { Julio H. Pimienta } \\
\text { (2012). }\end{array}$ & $\begin{array}{l}\text { Philippe Perrenoud } \\
\text { (2007). }\end{array}$ \\
\hline & España & España & México & Francia \\
\hline 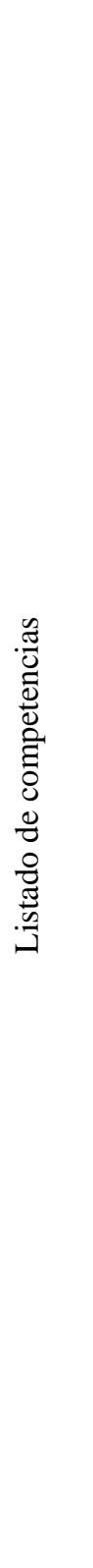 & 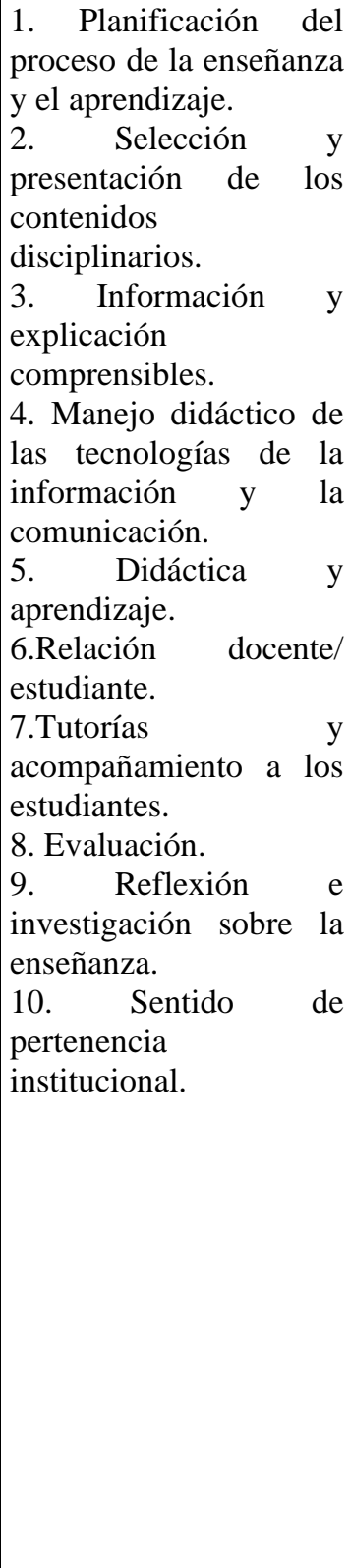 & 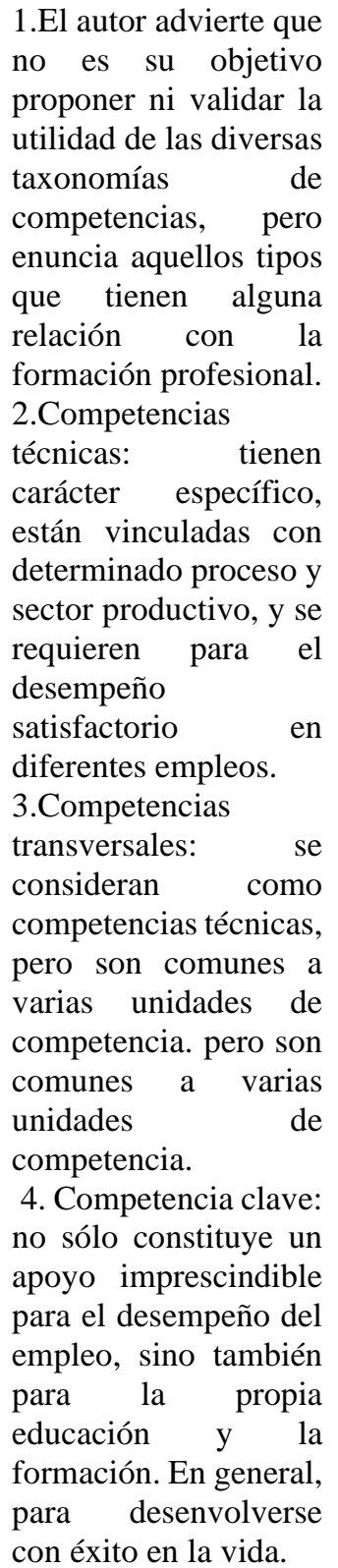 & $\begin{array}{l}\text { 1.Organizar y animar } \\
\text { situaciones } \\
\text { aprendizaje. } \\
\text { 2.Gestionar da } \\
\text { progresión de los } \\
\text { aprendizajes. } \\
\text { 3.Elaborar y hacer } \\
\text { evolucionar } \\
\text { dispositivos } \\
\text { diferenciación. } \\
\text { 4. Implicar a los } \\
\text { alumnos en sus } \\
\text { aprendizajes y en su } \\
\text { trabajo. } \\
\text { 5. Trabajar en equipo. } \\
\text { 6. Participar en la } \\
\text { gestión de la escuela. } \\
\text { 7. Informar e implicar } \\
\text { a los padres. } \\
\text { 8.Utilizar las nuevas } \\
\text { tecnologías. } \\
\text { 9. Afrontar los deberes } \\
\text { y los dilemas éticos de } \\
\text { la profesión. } \\
\text { 10.Organizar la propia } \\
\text { formación continua. }\end{array}$ & $\begin{array}{lr}\text { 1. Organizar y animar } \\
\text { situaciones } & \text { de } \\
\text { aprendizaje. } & 2 . \\
\text { Gestionar } & \text { la } \\
\text { progresión de } & \text { los } \\
\text { aprendizajes. } & 3 . \\
\text { Elaborar y hacer } \\
\text { evolucionar } \\
\text { dispositivos } \\
\text { diferenciación. } \\
\text { Implicar a los alumnos } \\
\text { en su aprendizaje. } 5 . \\
\text { Trabajar en equipo. } 6 . \\
\text { Participar en la gestión } \\
\text { de la escuela. } 7 . \\
\text { Informar e implicar a } \\
\text { los padres. 8. Utilizar } \\
\text { las nuevas tecnologías. } \\
\text { 9. Afrontar los deberes } \\
\text { y los dilemas éticos de } \\
\text { la profesión. } 10 . \\
\text { Organizar la propia } \\
\text { formación continua. }\end{array}$ \\
\hline
\end{tabular}




\section{DESARROLLO}

La presente propuesta se integra a partir de la valoración del trayecto profesional-formativo, presente-pasado, de 20 profesores, noveles y en activo del nivel superior y medio superior del IPN, y de diferentes áreas disciplinares; tales como ciencias de la ingeniería, administración, biología, arquitectura, medicina, lenguas extranjeras para así conformar un proyecto formativo en competencias didácticopedagógicos, presente-futuro, a partir de la reflexión personal y colectiva de la profesionalización docente en el contexto del IPN, a través de la recuperación e intercambio cuantitativo-descriptivo de experiencias de todos y cada uno de los profesores participantes, se analizaron los mecanismos de inserción en la docencia, las razones por las que se eligió la docencia, las trayectorias profesionales docentes, los estadios de carrera y los campos de actualización docente, para lo cual cada uno de estos hallazgos fue comparado a partir del marco jurídico del Instituto. La información se integró a través de una serie de foros que los autores del presente trabajo abrieron en la plataforma Moodle, la cual se usa en impartición de cursos y diplomados por la Coordinación General de Formación e Innovación Educativa (CGFIE), con preguntas ejes: Inicio en la docencia, Trayectoria docente, Permanencia en la Docencia y Formación Docente.

\section{RESULTADOS}

\subsection{INICIO EN LA DOCENCIA.}

Del análisis de la información recabada en las diferentes foros virtuales que los autores del trabajo abrieron, muestra que los docentes nos insertamos al IPN por la vía relacional a través de una invitación, por una recomendación, por la ayuda de un amigo; o sea que las relaciones sociales constituyen la principal forma de inserción; pero, el porcentaje restante confeso que se insertó por mecanismos de mercado, siendo la entrega de currículum directamente en la institución educativa la opción más utilizada. En cuanto las razones por las que elegimos la docencia, fueron por no encontrar empleo relacionado con la formación original, para completar el gasto familiar y estabilidad laboral. No obstante, hubimos profesores que afirmamos estar en la docencia por vocación.

\subsection{TRAYECTORIA DOCENTE.}

En lo que concierne a las trayectorias profesionales docentes, se halló la mayoría, siempre se ha desarrollado en la docencia y sólo ha tenido un empleo, otra es que inició en un puesto administrativo en su primer empleo y después se insertó en la docencia en su segundo empleo y primer empleo un puesto relacionado con su profesión y después se insertó en la docencia. 


\subsection{PERMANENCIA EN LA DOCENCIA.}

Para con los estadios de carrera, la mayoría confeso estar situada con bastantes años de experiencia profesional en educación, mientras que otros se encuentran en el estadio avanzado próximo al retiro y sólo unos pocos se ubican en el estadio inicial de su carrera. En cuanto a la edad, los profesores tenemos entre 40 y 60 años, muchos profesores experimentan con el manejo de TIC en el aula, de nuevas estrategias de aprendizaje, de materiales didácticos y de estrategias de evaluación. Los docentes denotan tener una actitud de innovación y cambio del repertorio pedagógico acumulado durante años, en algunos casos, relatamos tener una actitud de autoevaluación y replanteamiento del futuro de nuestra enseñanza.

\subsection{FORMACIÓN DOCENTE.}

Acerca de la actualización docente, la mayoría relatamos actualizarnos constantemente en áreas como docencia, desarrollo personal y profesional. El elevado porcentaje actualización en la docencia evidencia nuestra falta de formación, debido a que muchos de los profesores que nos desempeñamos en el nivel medio superior y superior, provinimos de disciplinas ajenas a la enseñanza. Esta situación se hace evidente en la fase de experimentación y diversificación, por lo que los maestros realizamos estudios de posgrado y tomamos cursos para cubrir nuestras necesidades de formación. En la formación personal nos inclinamos un poco más por los cursos, aunque pocos en el campo profesional, tendemos a tomar posgrados. De los diferentes relatos se nota que Existe una variedad de oportunidades de formación y actualización como las estadías técnicas, años sabáticos, becas comisión para realizar estudios de posgrado (maestría o doctorado); no obstante, sólo pueden tener acceso a ellas los docentes de medio tiempo, tres cuartos de tiempo y tiempo completo, por lo que los docentes de asignatura deben buscar otras alternativas para su desarrollo profesional docente.

\subsection{DISEÑO DE UNA PROPUESTA DE TRAYECTORIA INSTITUCIONAL PARA LA PROFESIONALIZACIÓN DOCENTE}

Con base en lo relatado en el inciso anterior y consiente que en la Educación Media Superior y Superior del IPN la formación integral de los estudiantes es un elemento clave de su misión; sin embargo, su concreción requiere que los cuadros profesionales encargados de conducir y guiar los procesos de enseñanza-aprendizaje cuenten con los espacios académicos de reflexión, teorización y acercamiento a la realidad práctica del trabajo docente, que les permitan contar con las herramientas y competencias disciplinarias, didáctico-pedagógicas, genéricas y humanas, necesarias para afrontar con éxito el reto de ser profesor en procesos de formación integral a partir de metodologías centradas en el aprendizaje. Propongo que las diferentes acciones de todo programa de profesionalización docente (simposios, 
conferencias, foros, cursos, talleres, seminarios, encuentros y diplomados, entre otras), deben estar estructuradas a partir de dos dimensiones: a) Profesional, cuyo objetivo es brindar al docente las competencias profesionales necesarias (disciplinarias, didáctico-pedagógicas y genéricas), para garantizar su óptimo desempeño profesional; y, b) Personal, orientada a promover y fortalecer el crecimiento en sus ámbitos personal, social, psicológico, físico, ético y ciudadano. Cada una de estas dimensiones debe de estar integrada por áreas específicas de desarrollo, tal y como se describe a continuación.

Profesional

a) Disciplinar: Se refiere a la formación de profesores en el área o conjunto de áreas del saber, de formación de origen o del desempeño académico actual, partiendo de lo que sucede en la sociedad del conocimiento, en la que los conocimientos representan un constante cambio y los que fueron adquiridos en la etapa de formación inicial tienen fecha de caducidad, y no son suficientes para toda una vida profesional activa; es entonces cuando se vuelve indispensable que los profesionistas adquieran una actitud de permanente aprendizaje frente a tales retos.

b) Didáctica general y de las disciplinas: Formación que tiene por objeto la habilitación en la organización y orientación de situaciones de enseñanza-aprendizaje de carácter instructivo, tendientes a la formación del individuo en estrecha dependencia de su educación integral. Capacitarse en las didácticas de las disciplinas o específicas, que estudian los modos de enseñar y aprender las distintas variantes de las artes, ciencias, lenguajes y tecnologías, que compartan espacios importantes de sus fundamentos epistemológicos, campos de actuación profesional y desarrollo de la investigación. Habilitar sobre el conocimiento didáctico del contenido, que aparece como un elemento central del conocimiento del profesor y representa la combinación adecuada entre el conocimiento de la materia a enseñar y el conocimiento referido a cómo enseñarla. Plantea la necesidad de que los profesores en formación adquieran un conocimiento experto del contenido a enseñar, para que puedan desarrollar una enseñanza que propicie la comprensión de los alumnos.

Curricular: Desarrollar competencias a partir del significado del currículo, al cual se le considerará como un sistema desarrollado para trabajar con personas y el proceso de organización de personal y sus procedimientos de implementación; su esencia incluye dos entidades interrelacionadas: en primer lugar, el registro escrito del consenso grupal acerca de los métodos, materiales, alcances y desarrollo del proceso educativo; y en segundo lugar, las subjetividades del maestro y del estudiante aparecen como la suma de experiencias y orientaciones de la acción que cada uno ha interpretado para sí como resultado de su vida interactiva y su aprendizaje conjunto en la escuela. Para ello, es importante que el profesor no sea sólo un actor importante en la aplicación de programas curriculares, sino que se involucre también en su diseño. 
Gestión del conocimiento: capacitar al docente en diferenciar información de conocimiento; donde la información es un conjunto de datos que presenta cierto nivel de asociación entre sus elementos, responde a cuestiones de quién, cuál, cuándo, dónde, cuántos, así, cuando la información es utilizada y puesta en contexto, se transforma en conocimiento. La gestión del conocimiento consiste en "saber cómo acceder a las informaciones, seleccionarlas, articularlas y aplicarlas a un determinado objetivo", ésta, aunque simple, nos sitúa en el contexto educativo aún no resuelto por muchos de los profesores; el uso de la tecnología en el aula y la gestión del autoaprendizaje.

Personal

Desarrollo personal: Forma en referencia a las múltiples facetas que conforman el desarrollo y la naturaleza de la persona, como: la integración consigo mismo y la sociedad, el mundo global y las tradiciones en que nacemos, lo que experimenta como absoluto o trascendente (religión); en el ámbito educativo refiere a la diversidad de puntos de vista, disciplinas o desde las perspectivas que se aproxima, observa, reflexiona y actúa la realidad.

Entonces la formación en esta propuesta se considera integral en la medida en que conciba al profesor en su totalidad y no sólo en su potencial cognoscitivo o en su capacidad para el quehacer técnicoprofesional. Su ámbito es el de una práctica educativa, que además de centrarse en la persona (docente) se orienta a cualificar su socialización para que pueda desarrollar la capacidad de auto-servirse del potencial de su espíritu, en el marco de la sociedad en que vive y pueda comprometerse con sentido histórico en transformarla mediante su labor cotidiana.

La propuesta de formación en el espacio tiempo de la profesionalización docente se propone organizarse en las siguientes etapas:

Iniciación docente. El profesor novel que se inserta a realizar las funciones docentes solo posee formación científica sobre la disciplina que explica, pero no dispone de los fundamentos pedagógicos para dirigir el proceso de enseñanza aprendizaje. Durante esta etapa se familiariza con el estudio de los documentos normativos de la educación superior, participa en la supervisión de actividades realizadas por profesores de experiencia, discutiendo con ellos los resultados de la observación; similar estrategia se desarrolla con los profesores noveles al ser visitados. Todas las actividades mencionadas son planificadas por el departamento docente al cual pertenece el profesor.

Adiestramiento docente. Esta etapa, fundamentalmente, se realiza a través del trabajo metodológico en los diferentes niveles organizativos en que está implicado el profesor Durante dicha etapa el profesor participa de forma activa en las diferentes actividades metodológicas concebidas por su departamento docente, siendo protagonista en la realización de clases abiertas, disertaciones de trabajos pedagógicos, visitas a profesores de experiencia con el propósito de mejorar la calidad de sus clases. 
Formaciones pedagógicas por niveles. A partir de un diagnóstico de necesidades de aprendizaje realizado, a los profesores, estos son ubicados en los distintos niveles de formación y consecuentemente, se estructuran en sistemas los diferentes postgrados a cursar. Para ello se toma en consideración las necesidades personales, sociales e institucionales. Los niveles de formación pedagógica previstos son: Básico: Incluye a los profesores que no han cursado estudios sobre la Didáctica de la Educación Superior. Mediante el curso básico de Pedagogía los profesores recibirán los conocimientos y habilidades esenciales sobre el desarrollo del proceso de enseñanza aprendizaje de la Educación Superior. Básico actualizado: Comprende la actualización de los profesores en los componentes del proceso de enseñanza aprendizaje superior y su aplicación práctica a los diferentes niveles organizativos. Los profesores se agrupan en un curso de actualización pedagógica. Profundización: Está concebido para los jefes de carrera, departamento, colectivo de años, disciplina y asignatura, así como para otros profesores y directivos que lo requieran. Estos profesores participan en el diplomado de didáctica y dirección de la Educación Superior. Especialización: Está destinado a la especialización en los contenidos de las Ciencias de la Educación vinculado a las necesidades del puesto laboral. Los profesores designados participan en cursos de especialización diseñados a tales efectos.

Formación académica investigativa. Está dirigida al perfeccionamiento continuo del docente de nivel medio superior y superior, dicha formación se da a lo largo de su vida profesional. Incluye actividades de auto superación, que realiza el profesor para sistematizar y profundizar los conocimientos obtenidos a través del sistema de formación pedagógica acreditado por el Instituto. Asimismo, se incorpora a todas aquellas actividades organizadas por la institución u otras para continuar elevando su desarrollo profesional y personal. Se ofrecen como alternativas para continuar su formación los programas de Maestría en Educación y Doctorados en Ciencias Pedagógicas y en Educación.

La propuesta con todos sus elementos se presenta en el organizador gráfico de la figura 1.

En cada uno de los niveles se brinda especial atención a la participación en eventos pedagógicos para potenciar el intercambio académico con otros profesores y contraponer sus ideas, creencias, opiniones sobre el perfeccionamiento de la labor docente de la Educación Media Superior y Superior del IPN. Las cuatro etapas referidas están íntimamente vinculadas y van proyectando el trabajo de investigación de los profesores alrededor de su propio proceso. Desde esta perspectiva, necesariamente el profesor para lograr una formación pedagógica, según las exigencias de la Educación Media Superior y Superior actual, no tiene que transitar por cada una de las 3 primeras etapas señaladas, esto estará en dependencia del nivel de desarrollo profesional y pedagógico que ha alcanzado a través de su práctica docente. 
La propuesta de formación pedagógica se caracteriza por: El uso del diagnóstico pedagógico. Se parte de la identificación de las necesidades de aprendizajes personales, sociales e institucionales para organizar el sistema de cursos que se ofertan. El carácter participativo de los profesores. Los profesores participan en la elaboración de los programas que se ofertan con vistas a responder a las necesidades de aprendizajes señaladas.

Fig.1. Organizador gráfico de la vinculación entre competencias docentes y áreas de formación, delineando las posibles trayectorias para el desarrollo profesional docente en el Nivel Medio Superior y Nivel Superior del IPN.



\section{CONCLUSIONES}

Con base a la experiencia de los docentes autores de la presente propuesta, en la profesionalización docente en el IPN se concluye que:

Es innegable la importancia de la formación inicial de los profesores, a la hora de explicar los resultados de aprendizaje de los estudiantes. En este sentido, un programa de perfeccionamiento docente sobre la base de una insuficiente formación inicial no podrá alcanzar todos los objetivos que se ha planteado, pues deberá orientar sus esfuerzos a llenar vacíos para los cuales en muchas situaciones no tiene una propuesta adecuada. 
La propuesta que se ofrece tiene una fuerza de impacto enorme, pues genera una mística y lenguaje común, facilita el compartir experiencias pedagógicas enriquecedoras entre pares, favorece la priorización de metas y permite desarrollar una identidad compartida, todo lo que ha sido descrito como un factor importante en la efectividad.

Para que exista una real sustentabilidad de las mejoras alcanzadas, es necesario hacer partícipe a toda la comunidad educativa de cada unidad académica en la gestión de la aplicación y mantención de los cambios obtenidos. En este sentido es especialmente importante el rol de los equipos directivos de cada unidad académica y de las autoridades institucionales. Sin líderes pedagógicos activos y propositivos la mantención de logros es inviable.

Por otra parte, es necesario instalar en las unidades académicas un sistema de autoformación continua que asegure la formación de los docentes que se incorporen al sistema luego de finalizado el perfeccionamiento. La pertinencia de esta propuesta y, en general, de las propuestas que existen para mejorar la calidad de la educación a través del perfeccionamiento docente, están orientadas a dar una respuesta adecuada para escuelas tradicionales, y está bien que así sea.

Otras coincidencias de nuestras relatorías son, que cuando se inicia la carrera docente en el IPN, la mayoría, estábamos influenciados por la sucesión de referentes docentes positivos y negativos de cuando éramos estudiantes, además de aspectos personales, familiares, institucionales, contextuales y sociales que afectaron en el inicio de nuestra carrera docente; la mayoría éramos jóvenes, recién graduados, con alguna experiencia profesional pero sin experiencia docente, que accedimos a un puesto docente, ya sea como profesor adjunto o asociado, generalmente, sin ningún tipo de acompañamiento docente, que en el periodo de iniciación a la docencia, se nos presentaron una variedad de vivencias ambiguas y confusas, de preocupaciones y de ilusiones que nos generaban sentimientos de angustia, incertidumbre e inseguridad. Como profesores principiantes tuvimos que aprender durante la práctica cómo planear una clase, diseñar las actividades a trabajar en el aula, recurrir a estrategias didácticas y diseñar instrumentos de evaluación como cualquier profesor con experiencia en la docencia; por tal motivo, fue un período de tensiones y aprendizajes que nos fueron intensivos en contextos que nos eran desconocidos y durante el cual como profesores principiantes tuvimos que adquirir el conocimiento profesional, además de mantener un cierto equilibrio como personas. También coincidimos que, el trabajo en la docencia no era del todo desconocido, ya que nos habíamos desenvuelto en el aula desde nuestra etapa de estudiantes, por lo que conocíamos los procesos y las reglas de trabajo a través de la interacción con nuestros profesores, la mayoría de nosotros comenzó su labor con expectativas altas sobre la enseñanza. En cuanto a la formación inicial se resalta que la mayoría se capacita por motivación personal para mejorar nuestras habilidades técnicas a través de los diferentes programas de formación y 
actualización que ofrece el IPN, que fue un proceso gradual, ya que se desarrolló por medio de la acumulación de descubrimientos y aprendizajes que fueron individuales más que colectivos, obtenidos por medio de experiencias, donde cada uno avanzo de acuerdo a su ritmo, presentando diferentes niveles de madurez personal y profesional. En resumen, que el ingreso a la docencia se presenta por mecanismos relacionales y un gran número de docentes no cuenta con formación pedagógica; así mismo reconocen que desde el principio de su ingreso tuvieron posibilidades de desarrollo profesional si contaban, por lo menos, con una estabilidad laboral de una plaza de al menos medio tiempo.

Finalmente se concluye que, con la puesta en práctica de la presente "Propuesta de profesionalización docente para profesores de nivel superior y media superior en el IPN", acompañada de la observación y reflexión constante que permita realizar reajustes, modificaciones y convertirla en una acción críticamente informada., correctamente operada y evaluada en sus resultados se lograría, una mejora sustancial de la calidad de la práctica docente, en los niveles superior y medio superior del IPN. 


\section{BIBLIOGRAFÍA}

Instituto Politécnico Nacional (2003). UN NUEVO MODELO EDUCATIVO PARA EL IPN. México, IPN.

Desgagné, S. (2005). Récits exemplaires de practique enseignante. Analyse typologique. Québec: Presses de l’Université du Québec.

Diario Oficial de la Federación (DOF)-Secretaría de Educación Pública (SEP), “Acuerdo número 447 por el que se establecen las competencias docentes para quienes impartan educación media superior en la modalidad

escolarizada", DOF, México, 2008,enlínea:http://www.sep.gob.mx/work/models/sep1/Resource/7aa2c3f f-aab8-479f-ad93-db49d0a1108a/a447.pdf,consulta:9 de octubre de 2018.

Garduño R. (2004). Cuaderno de Autoevaluación de competencias docentes. Dirección General de Servicios Educativos del DF. Coordinación Sectorial de Educación Primaria. (En prensa)

hang, M.-L. (2009). Teacher emotion management in the classroom: Appraisals, regulation, and coping with emotions. Unpublished dissertation.

hang, M.-L. (2009). Teacher emotion management in the classroom: Appraisals, regulation, and coping with emotions. Unpublished dissertation.

hang, M.-L. (2009). Teacher emotion management in the classroom: Appraisals, regulation, and coping with emotions. Unpublished dissertation.

Chang, M.-L. (2009). Teacher emotion management in the classroom: Appraisals, regulation, and coping

with emotions. Unpublished dissertation.

Chang, M.-L. (2009). Teacher emotion management in the classroom: Appraisals, regulation, and coping

with emotions. Unpublished dissertation.

Chang, M.-L. (2009). Teacher emotion management in the classroom: Appraisals, regulation, and coping with emotions. Unpublished dissertation.

Perrenoud, Phillippe (2004). Desarrollar la práctica reflexiva en el oficio de enseñar. Profesionalización y razón pedagógica. Barcelona Graó

Roegiers, X. (2010). Pedagogía de la integración. Competencias e integración de los conocimientos en la enseñanza. San José, Costa Rica: Coordinación Educativa y Cultural Centroamericana y AECI. Colección IDER (Investigación y desarrollo educativo regional). 